\title{
Consentimiento informado. ¿'Requisito legal o ético?
}

\author{
Informed consent: Legal or ethical requirement? \\ Arturo Rafael Vázquez Guerrero, * Éctor Jaime Ramírez Barba, ** \\ Jorge Arturo Vázquez Reta, ${ }^{* * *}$ Fernanda Cota Gutiérrez, ${ }^{* * * *}$ \\ Jorge Arturo Gutiérrez Muñoz*****
}

\begin{abstract}
Palabras clave:
Consentimiento

informado, requisito

ético, requisito legal,

derecho sanitario.
\end{abstract}

Key words:

Informed consent, ethical requirement,

legal requirement, sanitary law.

* Lic. en Derecho y

Ciencias Sociales. Médico

Especialista en Salud

Pública, Cirugía General

y Cirugía Oncológica.

Candidato a Doctor en

Ciencias Médicas.

** Médico Especialista

en Salud Pública

y Cirugía General,

Doctor en Ciencias

Médicas, Doctor

en Administración

Pública. PhD. Academia

Mexicana de Cirugía.

Academia Nacional de

Medicina. Universidad

de Guanajuato.

*** Médico Especialista

en Cirugía General,

Cirugía del Aparato

Digestivo, Endoscopia

Gastrointestinal. Academia

Mexicana de Cirugía.

**** Médico Pasante de

Servicio Social. Instituto

de Ciencias Biomédicas,

Universidad Autónoma

de Ciudad Juárez.

Chihuahua, Chih.

***** Abogado, Maestro

en Derecho Político y

Administración Pública.

Consultor del Sistema

Penal Acusatorio. Instituto

Nacional de Ciencias

Penales (INACIPE).

Ciudad de México.

Instituto Mexicano

del Seguro Social.

Departamento de Cirugía

Digestiva y Endocrina.

Hospital San Lucas de

Monterrey, N.L., México.

Recibido: 15/04/2016

Aceptado: 30/10/2017

\section{RESUMEN}

El consentimiento informado es la institución, el proceso y el documento médico-legal y bioético más trascendente de la relación médico-paciente o prestador usuario de los servicios de salud. Su reglamentación se encuentra dispersa en cuerpos normativos de diferente naturaleza como la legislación civil, la Ley General de Salud, sus reglamentos y las Normas Oficiales Mexicanas. Además de las anteriores, la perfección ético-legal del consentimiento informado implica respetar sendos principios bioéticos como la beneficencia, no maleficencia, autonomía y justicia. ¿Es el consentimiento informado un requisito legal o ético de los actos jurídicos de la relación médico-paciente? Es la pregunta de la investigación. Por lo anterior, el objetivo del presente estudio radica en exponer el consentimiento informado en su triple esfera (médico, legal y bioética), sus diferentes expresiones en la legislación mexicana y comentar sus principales implicaciones bioéticas. La naturaleza jurídica de la relación médico-paciente es la de un contrato de prestación de servicios profesionales con derechos y obligaciones para las partes. El consentimiento es un requisito de existencia del anterior contrato. El consentimiento informado se define como el acuerdo de voluntades entre médico y paciente en el que el primero realiza una propuesta de plan médico o quirúrgico, informa riesgos, beneficios y solicita autorización para atender urgencias y contingencias derivadas del acto autorizado seguido de la aceptación y firma del paciente (o de su representante en caso necesario), médico y dos testigos. El CI debe respetar simultáneamente los requisitos, forma y contenido establecido por la legislación civil, sanitaria y los principios bioéticos de autonomía, beneficencia, no maleficencia y justicia para alcanzar su existencia y validez jurídica, así como su perfección ética y legal.
Informed consent is the institution, process and most important medical-legal and bioethical document of the doctor-patient or provider-user relationship of health services. Its regulations are scattered in different regulatory bodies of different nature such as civil legislation, the General Health Law, its Regulations and the Mexican Official Norms. In addition to the above, ethical-legal perfection of informed consent implies respect for bioethical principles such as beneficence, not maleficence, autonomy, and justice. Research question: Is informed consent a legal or ethical requirement for legal acts of the physician-patient relationship? Therefore, the objective of this document is to set forth informed consent in its triple sphere (medical, legal and bioethical), its different expressions in Mexican legislation and comment on its main bioethical implications. The legal nature of the patient medical relationship is that of a contract for the provision of professional services with rights and obligations for the parties. Consent is a prerequisite for the existence of the previous contract. Informed consent is defined as the agreement of will between physician and patient in which the former makes a medical or surgical plan proposal, reports risks, benefits and requests authorization to attend to emergencies and contingencies arising from the authorized act followed by the patient's (or his/her representative if necessary), physician and two witnesses'signature and acceptance. The informed consent must simultaneously respect the requirements, form and content established by civil and health legislation and the bioethical principles of autonomy, beneficence, nonmaleficence and justice in order to achieve its existence and legal validity, as well as its ethical and legal perfection.

\section{INTRODUCCIÓN}

$E^{\prime}$ consentimiento informado $(\mathrm{Cl})$ es el acto más importante de la relación médicopaciente debido a que plasma el momento en el que se formaliza el vínculo del cual derivan derechos y obligaciones para las partes. El con- sentimiento informado en salud conlleva una serie de antecedentes e implicaciones legales y bioéticas profundas y de gran amplitud. Por esta razón, con la finalidad de descubrir si el consentimiento informado es un requisito legal o ético de los actos jurídicos de la relación médicopaciente, expondremos la naturaleza jurídica de 
la relación médico-paciente y su naturaleza contractual de prestación de servicios profesionales. Acto seguido, estudiaremos el consentimiento como requisito de existencia del acto jurídico analizando los aspectos civiles del consentimiento lato sensu, para pasar a especificar nuestro análisis del consentimiento informado en el derecho sanitario. Una vez expuesta la parte jurídica dogmática del consentimiento en general y del consentimiento informado, examinaremos la legislación sanitaria en bioética.

Finalmente se integrará la legislación civil en materia de consentimiento y sanitaria en consentimiento informado a los principios generales de bioética para esclarecer su estrecha relación de complementariedad y correspondencia y definir el consentimiento informado como un requisito jurídico y ético de los actos jurídicos de la relación médico-paciente.

\section{NATURALEZA JURÍDICA DE LA RELACIÓN MÉDICO-PACIENTE}

\section{La relación médico-paciente como contrato de prestación de servicios profesionales}

Al emprender el objetivo de definir la naturaleza jurídica de la relación médico-paciente observamos la participación de dos humanos, que a partir del acuerdo de voluntades inician un intercambio de prestaciones y contraprestaciones. El médico-prestador y el paciente-usuario con derechos y obligaciones bilaterales y recíprocas.

Este contrato, del latín contractus, derivado a su vez del verbo contrahere significa reunir, lograr o concertar. El contrato, en sentido amplio, es un acto jurídico bilateral que nace del acuerdo de voluntades de dos o más personas y que produce consecuencias jurídicas (crear y transmitir derechos y obligaciones). Más adelante, al explorar la legislación civil federal encontramos el contrato de prestación de servicios profesionales regulado por el Código Civil Federal $^{1}$ (CCF Arts. 2606-2615) y el de las entidades federativas. El primero señala que es el contrato entre el que presta y el que recibe servicios profesionales, en el cual la retribución se fija por común acuerdo (CCF 2006). A continuación se exponen los principales atributos, características, derechos y obligaciones del contrato de prestación de servicios profesionales.
Retribución. El principio civilista de libertad de contratación estipula que la retribución la establecen las partes de común acuerdo. A falta de convenio, la retribución se determina de acuerdo con los siguientes criterios: i. la costumbre; ii. la importancia del trabajo o asunto; iii. la capacidad del que recibe el servicio y iv. la reputación profesional del prestador. (CCF 2606-2607).

Retribución y autorización para el ejercicio profesional. Aquéllos que ejerzan una profesión para la que la ley exija un título y no cuenten con éste, además de incurrir en sanciones y penas, no tendrán derecho a cobrar retribución por los servicios profesionales que hayan prestado (CCF 2608). Este artículo nos reenvía al Capítulo I "Profesionales, técnicos y auxiliares", del Título Cuarto "Recursos Humanos para los Servicios de Salud" de la LGS para lo relativo al ejercicio de las profesiones, técnicos, auxiliares y especialistas en la salud (LGS 78-83).

Gastos necesarios para la prestación de los servicios profesionales. En la prestación de servicios profesionales pueden incluirse las expensas necesarias para la prestación del servicio en el negocio o asunto convenido (CCF 2609).

Obligación de medios y de resultados. Este contrato genera en el médico la obligación de medios, no a la obligación de resultados (a menos de que se comprometa expresamente a esto) es decir, se obliga a actuar apegado a la lex artis, independientemente del resultado del asunto o caso. Dicho de otro modo, la obligación consiste en aplicar los conocimientos científicos y recursos técnicos necesarios y disponibles al servicio del paciente (CCF 2613). La ley no lo obliga a resultados.

Responsabilidad civil. El prestador de servicios profesionales sólo es responsable por negligencia, impericia o dolo (sin perjuicio de las sanciones o penas por incumplimiento de normativa de otra índole como la penal o administrativa) (CCF 2615).

Además de lo anterior, debemos señalar las siguientes obligaciones inherentes a este tipo de contrato. Deberes del médico: i. elaborar la historia clínica; ii. asistir al paciente (obligación de medios); iii. configurar un diagnóstico; iv. ofrecer un plan terapéutico; v. informar; vi. 
referir al paciente a otro especialista o establecimiento de acuerdo con el caso; vii. guardar la confidencialidad médica; viii. elaborar un certificado médico o resumen clínico previa solicitud, entre otros: Deberes del paciente: i. ser fidedigno, verídico y fiel en la comunicación con el médico; ii. adherencia a la terapia; iii. retribución o pago de honorarios, entre otros.

Por lo anterior, la naturaleza jurídica de la relación médico-paciente es contractual y se encuadra en el contrato de prestación de servicios profesionales.

\section{El consentimiento como requisito de existencia del acto jurídico (Aspectos civiles del consentimiento lato sensu)}

Existen numerosas definiciones del vocablo "consentimiento". Éste es el acuerdo de dos o más voluntades destinadas a producir consecuencias o fines de interés legal en la celebración de cualquier convenio o contrato. ${ }^{2}$ Asimismo, se define como la adhesión a la voluntad de otro o el concurso mutuo de la voluntad de las partes sobre un hecho que aprueban con pleno conocimiento. ${ }^{3}$ El consentimiento es un requisito de existencia del contrato, es decir sin consentimiento no hay contrato. Para entender el consentimiento informado en medicina es imperioso comprenderlo como concepto civil y requisito de existencia de los actos jurídicos que responda a la pregunta que motiva el presente ensayo "El consentimiento informado es un requisito legal o ético". De tal modo, los requisitos de los contratos son de dos tipos: I. de existencia y II. de validez.

\section{Requisitos de existencia (requisito esen-} cial o sine qua non). i. el consentimiento: (compuesto por la oferta y aceptación). ii. el objeto posible: el objeto directo del contrato es crear y transferir derechos y obligaciones, el objeto indirecto puede consistir en acciones de dar, hacer o no hacer (v.g. dar un diagnóstico, hacer una hemitiroidectomía indicada, no hacer tiroidectomía total contraindicada para no hacer un daño -primum non nocere-). En resumen, para descubrir cuál es el objeto de un acto jurídico es preciso responder a la pregunta ¿A qué está obligado el médico o el paciente? considerando que el objeto posible debe serlo física y jurídicamente; iii. la solemnidad: la importancia económica o social de ciertos actos (v.g. en el derecho familiar se encuentra el matrimonio, la adopción, etc.) impone el requisito de celebrarlos con determinados ritos que condicionan su existencia.

II. Requisitos de validez (necesarios para producir efectos jurídicos plenos). i. capacidad de las partes: de goce y de ejercicio; ii. ausencia de vicios de la voluntad: a) el error (decisión que proviene de una creencia equivocada o concepto falso de la realidad, v.g. someterse a una cirugía desconociendo el verdadero riesgo o la falta de beneficio), b) el dolo (la decisión se obtiene o mantiene por engaños, v.g. mentir en el diagnóstico para llevar a cabo una cirugía), c) el temor o violencia (empleo de fuerza física o amenazas, d) la lesión (la desproporción exagerada de las prestaciones que las partes se deban recíprocamente por el acto jurídico); iii. cumplimiento de la forma legal; iv. la licitud en el objeto, motivo o fin.

El estudio de los requisitos de los actos jurídicos nos permite concluir que el consentimiento, dado su carácter de requisito de existencia, es una condición sine qua non para el nacimiento del acto jurídico, mientras que los de validez son necesarios para que el acto jurídico produzca efectos jurídicos plenos. Por lo tanto, deberá cumplir con los elementos de existencia y validez, so pena de nulidad o invalidez.

\section{El consentimiento informado en el derecho sanitario}

Ya desarrollada la naturaleza civil del consentimiento lato sensu, procedemos a enlazar nuestro objeto de estudio profundizando en el análisis del consentimiento informado en la legislación sanitaria. Los puntos más relevantes son conocer las normas mexicanas que lo contienen, la definición de carta de consentimiento informado, el contenido de éstas, la serie de actos mínimos que requieren $\mathrm{Cl}$ y por último la interpretación de la Suprema Corte de Justicia de la Nación sobre esta importante institución. 
Las disposiciones legales sobre el consentimiento informado se encuentran dispersas por materia en los siguientes cuerpos normativos: i. Ley General de Salud (LGS); ${ }^{4}$ ii. Reglamento de la Ley General de Salud en materia de prestación de servicios de atención médica (R-LGS-AM); ${ }^{5}$ iii. Reglamento de la Ley General de Salud en materia de investigación para la salud (R-LGS-IS); ${ }^{6}$ iv. Reglamento de la Ley General de Salud en materia de trasplantes (R-LGS-MT); ${ }^{7}$ v. Norma Oficial Mexicana del Expediente Clínico (NOM EC). ${ }^{8}$

\section{Definición de consentimiento informa-} do. El acuerdo de voluntades entre médico y paciente en el que el primero realiza una propuesta de plan médico o quirúrgico, informa riesgos, beneficios y solicita autorización para atender urgencias y contingencias derivadas del acto autorizado seguido de la aceptación y firma del paciente (o de su representante en caso necesario), médico y dos testigos.

Carta de consentimiento informado. El(los) documento(s) escrito(s) signado(s) por el paciente o su representante legal o familiar más cercano en vínculo, mediante los cuales se acepta un procedimiento médico o quirúrgico con fines diagnósticos, terapéuticos, rehabilitatorios, paliativos o de investigación, una vez que se ha recibido información de los riesgos y beneficios esperados para el paciente (NOM EC 4.2).

Contenido de las cartas de consentimiento informado. Éstas deberán contener los siguientes: i. nombre de la institución y establecimiento (razón o denominación social); ii. título del documento; iii. lugar y fecha; iv. acto autorizado con riesgos y beneficios esperados; v. autorización para atender contingencias y urgencias derivadas del acto autorizado atendiendo al principio de libertad prescriptiva; vi. nombre completo y firma del paciente (o del familiar, tutor o representante si el estado de salud lo permite); vii. nombre completo y firma del médico que informa y recaba aceptación para el acto específico. Nombre del médico tratante en su caso; viii. nombre y firma de dos testigos (NOM EC 10.1.1).

Actos mínimos que requieren $\mathrm{Cl}$ : i. ingreso hospitalario; ii. procedimientos de cirugía mayor; iii. procedimientos que requieren anestesia general o regional; iv. salpingoclasia y vasectomía; v. donación de órganos, tejidos y trasplantes; vi. investigación clínica en seres humanos; vii. necropsia hospitalaria; viii. procedimientos diagnósticos y terapéuticos considerados por el médico como de alto riesgo; ix. cualquier procedimiento que entrañe mutilación; x. en casos en que el médico lo estime pertinente sin necesidad de formato especial (NOM EC 10.1.2).

Asimismo, la LGS y los reglamentos contienen una larga serie de situaciones específicas que requieren $\mathrm{Cl}$; sin embargo, conviene clasificarlas en las siguientes: a) según la materia sanitaria: prestación de servicios médicos, investigación, trasplantes; b) según la situación clínica: situaciones clínicas-quirúrgicas generales, situaciones específicas (urgencias, egreso voluntario, anticoncepción); c) según el tipo de paciente: derechohabiente del Sistema de Protección Social en Salud, enfermos en situación terminal, donador, trastorno mental; d) según la naturaleza del acto: quirúrgicos, anestésicos, de control natal, investigación post mortem, etc.; e) según el objetivo del acto: diagnóstico, terapéutico, paliativo, etc. f) según el que acepta: capaz, incapaz, sustituto o subrogado (v.g. dos médicos en urgencias, comité de bioética).

Por la importancia y frecuencia del $\mathrm{Cl}$ en ciertas situaciones, hemos elegido las siguientes para ampliar el comentario y realizar una serie de consideraciones: I. Urgencias; II. Ingreso hospitalario; III. Investigación.

I. Urgencias. La urgencia es todo problema médico-quirúrgico agudo que pone en peligro la vida, un órgano o una función y que requiere atención inmediata (R-LGS-AM 72). En caso de urgencia o de incapacidad del paciente, el consentimiento puede ser suscrito por el familiar o representante legal. Si en el contexto de una urgencia se está en presencia de un paciente incapaz de otorgar el consentimiento, con familiares ausentes, el reglamento faculta a los médicos a realizar algún procedimiento terapéutico si por lo menos dos de ellos dejan constancia escrita de su necesidad en el expediente clínico (R-LGS-AM 81). 
En este caso no es ético ni legal (en ausencia de familiares o representantes legales) dejar al paciente sin la atención requerida o retardar la misma.

II. Ingreso hospitalario. El ingreso hospitalario inicia la generación de derechos y obligaciones para las partes (médico-paciente, paciente-institución, médico-institución). En este momento el usuario debe signar autorización escrita para la práctica de los procedimientos médico-quirúrgicos necesarios (RLGSAM 80). En caso de urgencia o de incapacidad del paciente, el consentimiento puede ser suscrito por el familiar o representante legal. Posterior al ingreso, cada acto que ponga en riesgo al paciente debe ser precedido del consentimiento informado.

III. Investigación. En el contexto de investigación en salud, las normas, cantidad y calidad de la información intercambiada entre las partes se amplía para exponer claramente los objetivos de la experimentación y posibles consecuencias (positivas o negativas) para su salud (LGS 100-IV) con la salvaguarda del respeto a su dignidad y la protección de sus derechos y bienestar (R-LGS-IS 13).

Interpretación del Poder Judicial de la Federación (PJF) del $\mathrm{Cl}^{9}{ }^{9}$ El PJF publicó una tesis aislada en 2012 titulada "Consentimiento informado. Derecho fundamental de los pacientes", la cual no constituye jurisprudencia; sin embargo, aclara la visión del Máximo Tribunal en los siguientes aspectos: i. el $\mathrm{Cl}$ es un derecho fundamental de los pacientes; ii. el $\mathrm{Cl}$ es consecuencia de: a) derecho a la vida, b) derecho a la integridad física, c) derecho a la libertad en conciencia; iii. es derecho del paciente aceptar o rechazar el $\mathrm{Cl}$; iv. los requisitos para llevar a cabo lo propuesto por el Cl son: a) definir las características del acto a consentir, b) los riesgos; $v$. el objeto del $\mathrm{Cl}$ es realizar tratamientos o procedimientos; vi. el Cl no excluye la responsabilidad del médico o institución por negligencia. El análisis de la anterior tesis nos deja en claro la interpretación judicial del concepto de $\mathrm{Cl}$, su origen, sus principales elementos, relevancia y carácter de derecho fundamental para el paciente.

\section{DISPOSICIONES LEGALES SOBRE BIOÉTICA RELACIONADAS CON EL CONSENTIMIENTO INFORMADO}

\author{
Legislación sanitaria en bioética
}

En la legislación sanitaria encontramos diversas menciones y definiciones de bioética. En el decreto de creación de la Comisión Nacional de Bioética de 2005 se menciona como "el estudio de las cuestiones morales inherentes a la vida humana". ${ }^{10}$ Esta comisión es la autoridad encargada de establecer las políticas públicas en materia de bioética y ser garante de los derechos, seguridad, dignidad, bienestar de los usuarios. En diciembre de 2011 se reformó la LGS mediante adición del artículo 41 bis y reforma del 98. Esta modificación da operatividad y presencia hospitalaria a la comisión estableciendo los comités que habrán de concretar los fines y objetivos de promover la cultura bioética, fomentar la reflexión, deliberación, discusión multidisciplinaria y multisectorial de los temas vinculados con la salud humana, así como el desarrollo de la normativa bioética en tres áreas específicas: la atención médica, la investigación y la docencia. Es así como se determina que los establecimientos del Sistema Nacional de Salud contarán con los siguientes: I. Comité Hospitalario de Bioética y II. Comité de Ética en Investigación. ${ }^{11}$

I. Comité Hospitalario de Bioética. Su ámbito de acción es la práctica clínica y se cuenta con 459 a nivel nacional. Es un órgano autónomo, institucional, interdisciplinario, plural y de carácter consultivo para la resolución de los problemas derivados de la atención médica así como para el análisis, discusión y apoyo en la toma de decisiones respecto a los problemas bioéticos que se presenten en la práctica clínica o en la docencia. También contará con la función de elaborar lineamientos y guías éticas institucionales para la atención y la docencia médica y promover la educación bioética. (LGS 41 bis, Acuerdo Cuarta). ${ }^{12}$

II. Comité de Ética en Investigación. Su ámbito de acción es la investigación en salud y existen 335 a nivel nacional. Es un órgano autónomo, institucional, interdisciplinario, 
plural y de carácter consultivo para evaluar y dictaminar los protocolos de investigación en seres humanos. También tiene la función de formular y dar seguimiento a las recomendaciones éticas, elaborar lineamientos y guías éticas institucionales. (LGS 41 bis. Acuerdo Cuarta). ${ }^{13}$

\section{Los principios bioéticos en la legislación sanitaria y su relación con el CI}

El decreto de creación de la comisión enuncia de modo general los siguientes principios bioéticos (autonomía, beneficencia, no maleficencia, justicia y protección), sin hacer referencia expresa a su definición, ni mucho menos a sus alcances. La meta de la siguiente disertación es desentrañar la relación de cada uno de estos principios con el consentimiento informado.

Principio de autonomía y relación con el CI. En bioética, la autonomía es la capacidad para determinar libremente su pensamiento y conducta (derecho de autodeterminación). La autonomía requiere capacidad para tomar decisiones y habilidad para poner en práctica sus elecciones. Ahora bien, en el ámbito jurídicofilosófico este principio bioético encuentra su correspondiente en el principio de autonomía de la voluntad que atribuye a las personas un ámbito de libertad dentro del cual pueden regular sus propios intereses. Este dogma de la autonomía de la voluntad es enteramente aplicable al consentimiento informado, en el cual los autores del acto jurídico tienen la libertad de aceptar o rechazar su celebración y definir sin presiones y con la información suficiente el contenido y alcances en el caso concreto (v.g. riesgos, beneficios, modo de atender urgencias y contingencias para la cirugía programada: tiroidectomía total cuyo riesgo es el trauma quirúrgico a los nervios laríngeos recurrentes con parálisis y obstrucción aérea en el que el modo de atender la urgencia sería la traqueostomía inmediata).

Principio de beneficencia y relación con el Cl. Este principio bioético consiste en promover el bienestar o el mejor interés de una persona, mismo que encontramos como finalidad del derecho a la protección de la salud, "el derecho a la protección de la salud tiene las siguientes finalidades: El bienestar físico y mental de la persona para contribuir al ejercicio pleno de sus capacidades" (LGS 2-II) y como requisito de contenido mínimo de la carta de consentimiento informado de señalar los riesgos y beneficios esperados del acto médico autorizado (NOM EC 10.1.1).

Principio de no maleficencia y relación con el Cl. Este gran principio bioético prohíbe la generación de actos con consecuencias francamente negativas con evidente desequilibrio en detrimento del beneficio. Está ligado al primum non nocere, que además de ser el principio más valioso del legado hipocrático, establece categóricamente el buen inicio de la relación médico-paciente. Ante todo y primero que nada, no dañar. El consentimiento informado debe establecer en su contenido el tratamiento a las consecuencias negativas del acto autorizado. Por ello en el $\mathrm{Cl}$ debe estar señalada la autorización para atender las contingencias y urgencias que deriven del acto consentido, mismas que el médico deberá tratar con la libertad prescriptiva que le asiste. Ahora bien, esta libertad prescriptiva del médico en el consentimiento informado es interpretada por la Suprema Corte de Justicia de la Nación, de cuya tesis aislada podemos extraer los siguientes elementos: ${ }^{14} \mathrm{i}$. la libertad prescriptiva es parte del derecho al trabajo de los médicos establecido en el artículo $5^{\circ}$ constitucional; ii. es un principio científico y ético orientador de la práctica de la profesión médica; iii. este principio otorga discrecionalidad (no arbitrariedad) para actuar con fundamento en la ciencia actual; iv. su fin debe ser siempre buscar el beneficio del paciente (beneficencia); v. el principio debe considerar las circunstancias del caso concreto.

Como se aprecia, éste y el anterior principio están íntimamente relacionados con el imperativo de acercarse al bien y mantenerse lejos del mal, paradigmas que la bioética y el derecho comparten.

Principio de justicia y relación con el CI. Del latín iustitia, que a su vez proviene de ius, que significa lo justo. Para entender justicia debemos evocar la concepción de Ulpiano, de Aristóteles y la clasificación tripartita de justicia como criterio racional de lo justo y lo injusto. i. Ulpiano: para éste, justicia es la constante 
y perpetua voluntad de dar a cada quien lo suyo; ii. Aristóteles: para él, la justicia consiste en dar un tratamiento igual a los iguales y desigual a los desiguales; iii. la clasificación de justicia comprende: a) la justicia legal o general, b) la justicia distributiva, c) la justicia conmutativa. Esta última es la más aplicable al tema de la relación médico-paciente, pues es la que rige el intercambio entre personas que se encuentran en un plano de igualdad (v.g. relación contractual médico-paciente), tal y como debe concebirse la relación médico-paciente moderna, una relación en la que ambos participan con el mismo nivel de compromiso, entusiasmo y corresponsabilidad hacia el fin de protección de la salud.

Principio de protección y relación con el CI. El principio de protección se asocia estrechamente al de subsidiariedad y se despliega con mayor amplitud en situaciones en las que el paciente se encuentra en estado de vulnerabilidad (v.g. menores de edad, incapaces, participantes de investigación, situaciones de urgencia). En este caso, al ser el consentimiento informado un derecho fundamental del paciente, queda siempre protegido por la previsión de reglas especiales que equilibran la debilidad o fragilidad que caracteriza estas situaciones.

\section{CONCLUSIÓN}

El consentimiento informado es el acuerdo de voluntades entre médico y paciente en el que el primero realiza una propuesta de plan médico o quirúrgico, informa riesgos, beneficios y solicita autorización para atender urgencias y contingencias derivadas del acto autorizado. Posteriormente, a través de la deliberación libre de presiones y vicios del consentimiento se da la aceptación mediante la firma del paciente, del familiar o representante en su caso, sin soslayar el requisito del nombre y firma de dos testigos.

El consentimiento lato sensu es requisito de existencia del acto jurídico que formaliza la relación médico-paciente y lo convierte en una relación contractual a través del contrato de prestación de servicios profesionales. Lo anterior fundamenta al consentimiento informado como requisito legal sine qua non para la existencia del acto jurídico.
El conocer, considerar y respetar los principios generales de la bioética al momento de proponer, deliberar y aceptar el consentimiento informado determina su calidad de requisito ético.

Por lo anterior podemos concluir que el consentimiento informado es simultáneamente requisito legal ético de los actos jurídicos de la relación médico-paciente, siendo una institución primordialmente jurídica con profundas implicaciones bioéticas.

\section{REFERENCIAS}

1. Presidente-de-la-República. Código Civil Federal 1928. Diario Oficial de la Federación. México D.F. [Última reforma 24-12-13].

2. Ackerman J. Consentimiento. En: Fix-Zamudio $\mathrm{H}$. Diccionario Jurídico Mexicano. Ed. Histórica. México D.F.: Porrúa; 2011. p. 779-782.

3. Escriche J. Diccionario Razonado de Legislación y Jurisprudencia. Ed. Librería de Rosa, Bouret y C. Paris: 1851.

4. Presidente-de-la-República. Ley General de Salud. Diario Oficial de la Federación. www.dof.gob.mx 0702-1984 [Última reforma 20-04-2015].

5. Presidente-de-la-República. Reglamento de la Ley General de Salud en materia de prestación de servicios de atención médica. Diario Oficial de la Federación www.dof.gob.mx 14-05-1986 [última reforma 24-03-2014].

6. Presidente-de-la-República. Reglamento de la Ley General de Salud en materia de investigación en salud. Diario Oficial de la Federación. www.dof.gob. mx 06-01-1987 [última reforma 02-04-2014].

7. Presidente-de-la-República. Reglamento de la Ley General de Salud en materia de trasplantes. Diario Oficial de la Federación. www.dof.gob.mx 26-03-2014 [Sin reformas].

8. Presidente-de-la-República. Norma Oficial Mexicana NOM-004-SSA3-2012, Del expediente clínico. Diario Oficial de la Federación. www.dof.gob.mx 15-10-2012 [Sin reformas].

9. Poder Judicial de la Federación. El consentimiento informado. Derecho fundamental de los pacientes. Semanario Judicial de la Federación y su Gaceta. Tesis: 1a. XLIII/2012 (10a.). Libro XI, Agosto de 2012, Tomo 1. Pág. 478. Tesis Aislada (Constitucional).

10. Presidente-de-la-República. Decreto por el que se crea el órgano desconcentrado denominado Comisión Nacional de Bioética. Diario Oficial de la Federación www.dof.gob 07-09-2005.

11. Presidente-de-la-República. Decreto por el que se adiciona el artículo 41 Bis y se reforma el artículo 98 de la Ley General de Salud. Diario Oficial de la Federación www.dof.gob.mx 14-12-2011.

12. Presidente-de-la-República. Acuerdo por el que se emiten las Disposiciones Generales para la Integración y Funcionamiento de los Comités Hospitalarios de Bioética y se establecen las unidades hospitalarias que deben contar con ellos, de conformidad con los 
criterios establecidos por la Comisión Nacional de Bioética. Diario Oficial de la Federación www.dof. gob.mx 31-10-12.

13. Presidente-de-la-República. Acuerdo por el que se emiten las Disposiciones Generales para la Integración y Funcionamiento de los Comités de Ética en Investigación y se establecen las unidades hospitalarias que deben contar con ellos, de conformidad con los criterios establecidos por la Comisión Nacional de Bioética. Diario Oficial de la Federación www.dof. gob.mx 31-10-2012.

14. Poder Judicial de la Federación. Libertad prescriptiva del médico. Parte integradora del derecho al trabajo de los médicos. Semanario Judicial de la Federación y su Gaceta. Tesis: 1a. XXII/2013 (10a.). Primera Sala. Décima Época. Libro XVI, Enero de 2013, Pág. 637 Tesis aislada.

\section{Correspondencia:}

Arturo Rafael Vázquez Guerrero

Calle Churubusco Núm. 246,

Col. Cumbres, $2^{\circ}$ sector, 64610,

Monterrey, N.L., México.

Cel. 8182598082

E-mail: doctorarturovazquez@gmail.com 